\title{
Universal amplitude ratios for scaling corrections on Ising strips with fixed boundary conditions.
}

\author{
N. Sh. Izmailian* \\ Institute of Physics, Academia Sinica, \\ Nankang, Taipei 11529, Taiwan and \\ Yerevan Physics Institute, Alikhanian Brothers 2, 375036 Yerevan, Armenia
}

(Dated: July 30, 2018)

\begin{abstract}
We study the (analytic) finite-size corrections in the Ising model on the strip with fixed (+-) boundary conditions. We find that subdominant finite-size corrections to scaling should be to the form $a_{k} / N^{2 k-1}$ for the free energy $f_{N}$ and $b_{k}^{(n)} / N^{2 k-1}$ for inverse correlation length $\xi_{n}^{-1}$, with integer value of $k$. We investigate the set $\left\{a_{k}, b_{k}^{(n)}\right\}$ by exact evaluation and their changes upon varying anisotropy of coupling. We find that the amplitude ratios $b_{k}^{(n)} / a_{k}$ remain constant upon varying coupling anisotropy. Such universal behavior are correctly reproduced by the conformal perturbative approach.

PACS numbers: $05.50+\mathrm{q}, 75.10-\mathrm{b}$
\end{abstract}

*Electronic address: izmailan@phys.sinica.edu.tw 


\section{INTRODUCTION}

Finite-size scaling and corrections for critical systems have attracted much attention in recent decades. Although many theoretical results are now known about the critical exponents and universal relations among the leading critical amplitudes, not much information is available on ratios among the amplitudes in finite-size correction terms [2]. New universal amplitude ratios have been recently presented for the Ising model [3-6]. Consider an Ising ferromagnet on an $N \times M$ lattice. If $\Lambda_{0}>\Lambda_{1}>\Lambda_{2}>\Lambda_{3}>\ldots$ are the eigenvalues of the transfer matrix (TM), in the limit $M \rightarrow \infty$ the free energy per spin, $f_{N}$, and the inverse longitudinal spin-spin correlation length, $\xi_{n}^{-1}$, are

$$
f_{N}=\frac{1}{\zeta N} \ln \Lambda_{0} \quad \text { and } \quad \xi_{n}^{-1}=\frac{1}{\zeta} \ln \left(\Lambda_{0} / \Lambda_{n}\right)
$$

Here $\zeta$ is a geometric factor, which is unity for the square lattice and, in triangular and honeycomb geometries (also for the square lattice when the TM progresses along the diagonal [7]), corrects for the fact that the physical length added upon each application of the TM differs from one lattice spacing [8].

At the critical point $T_{c}$ the asymptotic finite-size scaling behavior of the critical free energy $\left(f_{N}\right)$ and the inverse correlation lengths $\left(\xi_{n}^{-1}\right)$ of an infinitely long $2 \mathrm{D}$ strip of finite width $N$ has the form [9, 10]

$$
\begin{gathered}
\lim _{N \rightarrow \infty} N^{2}\left(f_{N}-f_{\infty}\right)-2 N f_{\text {surf }}=A, \\
\lim _{N \rightarrow \infty} N \xi_{n}^{-1}=D_{n},
\end{gathered}
$$

where $f_{\infty}$ is the bulk free energy, $f_{\text {surf }}$ is the surface free energy and $A$ and $D_{n}$ are the universal constants, but may depend on the boundary conditions. In some 2D geometries, the values of $A$ and $D_{n}$ are known [9-11], to be related to the conformal anomaly number $(c)$, the conformal weight of the ground state $(\Delta)$, and the scaling dimension of the $n$-th scaling field $\left(x_{n}\right)$ of the theory

$$
A=\pi \zeta\left(\frac{c}{24}-\Delta\right), \quad D_{n}=\pi \zeta x_{n},
$$

for strip geometry. Here $\zeta$ is anisotropy parameter. The principle of unitarity of the underlying field theory restricts through the Kac formula the possible values of $\mathrm{c}$ and for each value of c only permits a finite number of possible values of $\Delta$. For the $2 \mathrm{D}$ Ising model, we have $c=1 / 2$ and the only possible values are $\Delta=0,1 / 16,1 / 2$. 
For Ising universality class there are five different boundary universality classes: periodic, antiperiodic, free, fixed and mixed (mixture of the last two). Two of them: periodic and antiperiodic boundary universal classes are in cylinder geometry and three boundary universal classes: free, fixed (+-) and mixed in strip geometry. For fixed $(+-)$ boundary conditions the spins are fixed to the opposite values on two sides of the strip. The mixed boundary conditions corresponds to free boundary conditions on one side of the strip, and fixed boundary conditions on the other. In the terminology of surface critical phenomena these three boundary universal classes: free, fixed (+-) and mixed correspond to "ordinary", "extraordinary" and "special" surface critical behavior, respectively.

The highest conformal weight $\Delta$, and the scaling dimension $x_{n}$ depends on the boundary conditions and for fixed (+-) boundary conditions they given by

$$
\Delta=\frac{1}{2}, \quad x_{1}=1, \quad x_{2}=2, \quad x_{3}=3, \ldots, \quad x_{n}=n .
$$

Quite recently, Izmailian and $\mathrm{Hu}$ [3] studied the finite size correction terms for the free energy per spin and the inverse correlation lengths of critical $2 \mathrm{D}$ Ising models on $N \times \infty$ lattice and 1D quantum Ising chain with periodic boundary conditions. They obtained analytic expressions for the finite-size correction coefficients $a_{k}$ and $b_{k}^{(n)}$ in the expansions

$$
\begin{aligned}
N\left[f_{N}-f_{\infty}\right] & =f_{s}+\sum_{k=1}^{\infty} \frac{a_{k}}{N^{2 k-1}} \\
\xi_{n}^{-1} & =\sum_{k=1}^{\infty} \frac{b_{k}^{(n)}}{N^{2 k-1}}
\end{aligned}
$$

and find that although the finite-size correction coefficients $a_{k}, b_{k}^{(n)}$ (for $n=1,2$ ) themselves are non-universal (except for $a_{1}$ and $b_{1}^{(n)}$ ), the amplitude ratios for the coefficients of these series $b_{k}^{(n)} / a_{k}$ are universal.

Later that result has been extended for the quantum Ising chain for antiperiodic and free boundary conditions [4] and for the two-dimensional (2D) Ising models on $\mathcal{M} \times \infty$ lattice with the special boundary conditions studied by Brascamp and Kunz (BK) [5]. It was shown that Brascamp and Kunz (BK) boundary conditions [12] belong to the mixed boundary condition universality class although the mixed boundary condition and the BK boundary condition are different on one side of the long strip.

In the present paper we will present exact calculations for a set of universal amplitude ratios for the Ising model with fixed $(+-)$ boundary conditions. We obtain analytic equa- 


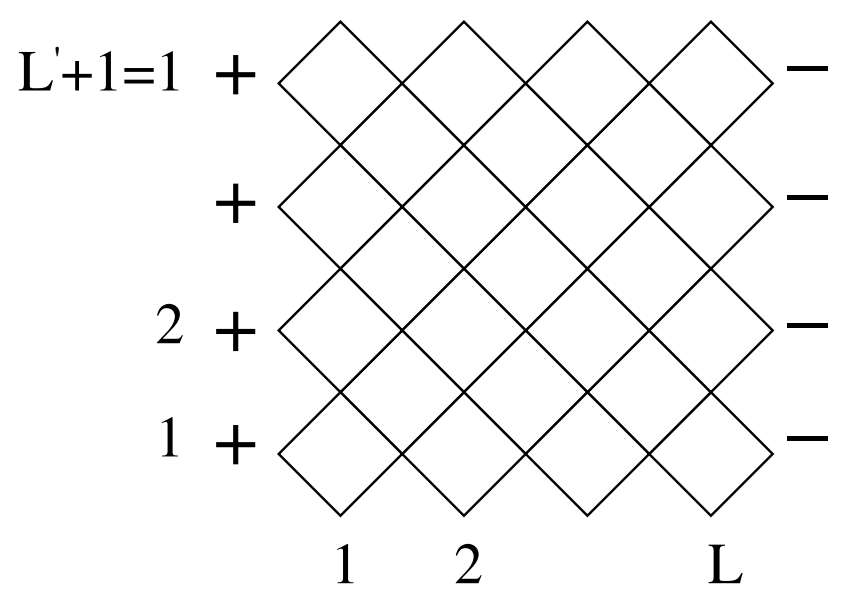

FIG. 1: The lattice $\mathcal{L}$

tions for $a_{k}$ and $b_{k}^{(n)}$ (for $n=1,2,3, \ldots$ ) in the expansions given by Eqs. (66) and (77) and find that amplitude ratios $b_{k}^{(n)} / a_{k}$ are universal. We will show that such universal behavior are correctly reproduced by the conformal perturbative approach.

\section{THE ISING MODEL ON CYLINDER}

The Ising model on a cylinder, with width $\mathrm{N}$ and height $\mathrm{M}$ and free, fixed and mixed boundary condition along diagonal was formulated in [7] in terms of a double-row transfer matrix $\mathrm{D}(\mathrm{u})$. They define the lattice $\mathcal{L}$ as a square lattice rotated by 45 degrees, in which the rows have alternately $L-1$ and $L$ faces. Vertically the lattice have columns of $L^{\prime}$ faces, and the periodic boundary conditions imposed in this direction by identifying the first and $\left(L^{\prime}+1\right)$ th rows of faces. The lattice $\mathcal{L}$ consists of $N$ (zigzagging) columns edges and $M$ (zigzagging) rows edges, respectively, and $N$ and $M$ are given by

$$
N=2 L \quad M=2 L^{\prime}
$$

The lattice $\mathcal{L}$ is shown in Fig. 1. We are concerned with the fixed $(+-)$ type of boundary conditions. For fixed $(+-)$ boundary conditions we choose the spins at the left and right boundaries of the lattice $\mathcal{L}$ to be +1 and -1 . 
The partition function is given by

$$
Z_{N M}=\sum_{\{\sigma\}} \exp \left(J \sum_{<i, j>} \sigma_{i} \sigma_{j}+K \sum_{<i, j>} \sigma_{i} \sigma_{j}\right)
$$

where we define the set of spins not fixed by the boundary conditions by $\{\sigma\}$. For fixed boundary conditions, the first sum is over edges in odd columns and the second sum over edges in even columns of the lattice.

Consider the critical Ising model on a cylindrical lattice of $N$ columns and $M$ rows as described previously. The finite-size partition function $Z_{N M}$ can be written as

$$
Z_{N M}(u)=\operatorname{Tr}\left(D(u)^{M}\right)=\sum_{n} e^{-2 M E_{n}(u)}
$$

where the sum is over all eigenvalues of a transfer matrix $D(u)$, written as $e^{-2 E_{n}(u)}$. Since we restrict ourselves to the critical Ising model, we have $\sinh (2 J) \sinh (2 K)=1$. This condition can be conveniently parameterized by introducing a so-called spectral parameter $u$, so that $\sinh (2 J)=\cot (2 u), \sinh (2 K)=\tan (2 u)$, with $0<u<\pi / 4$. The anisotropy parameter $\zeta$ related to the spectral parameter $u$ through

$$
\zeta=\sin 4 u
$$

For isotropic system $(K=J)$ we have $u=\pi / 8$ and $\zeta=1$.

For fixed $(+-)$ boundary conditions all eigenvalues have been determined in [7], for any finite value of $\mathrm{N}$. The energy $E_{n}$ associated with the eigenvalues of a transfer matrix $D(u)$ are given by

$$
E_{n}(u)=-\frac{1}{2} \ln \left\{2^{-N / 2} \prod_{k=1}^{N / 2}\left[\operatorname{cosec}\left(\frac{k-1 / 2}{N+1} \pi\right)+\mu_{k} \sin (4 u)\right]\right\}
$$

Using the identity

$$
\prod_{k=1}^{L} \sin \left(\frac{k-1 / 2}{2 L+1} \pi\right)=2^{-L}
$$

the Eq. (12) can be simplified to the following form

$$
E_{n}(u)=-\frac{1}{2} \sum_{k=1}^{N / 2} \ln \left[1+\mu_{k} \sin (4 u) \sin \left(\frac{k-1 / 2}{N+1} \pi\right)\right]
$$

where $\mu_{k}= \pm 1$. For fixed (+-) boundary conditions $\mu_{k}$ should satisfied the conditions $\prod_{k=1}^{L} \mu_{k}=-1$. This correctly yields $2^{L-1}$ eigenvalues for the fixed $(+-)$ boundary cases. 
The ground state $E_{0}$ correspond to all $\mu_{k}=1$ except $\mu_{1}=-1$ in Eq. (14). The exited levels $E_{n}$ are given by Eq. (14) with $\mu_{n}=-1$ and all other $\mu_{k}$ are equal to $1: \mu_{k}=1$ $(k \neq n)$. Thus for the critical free energy $\left(N f_{N}=-E_{0}\right)$ and the inverse correlation lengths $\left(\xi_{r}^{-1}=E_{r}-E_{0}\right)($ for $r=1,2, \ldots, n)$ we obtain

$$
\begin{aligned}
N f_{N} & =\sum_{k=1}^{\frac{N}{2}} \omega\left(\frac{k-1 / 2}{N+1} \pi\right)+\omega\left(-\frac{\pi}{2(N+1)}\right)-\omega\left(\frac{\pi}{2(N+1)}\right) \\
\xi_{1}^{-1} & =\omega\left(\frac{3 \pi}{2(N+1)}\right)-\omega\left(\frac{\pi}{2(N+1)}\right)+\omega\left(-\frac{\pi}{2(N+1)}\right)-\omega\left(-\frac{3 \pi}{2(N+1)}\right) \\
\xi_{2}^{-1} & =\omega\left(\frac{5 \pi}{2(N+1)}\right)-\omega\left(\frac{\pi}{2(N+1)}\right)+\omega\left(-\frac{\pi}{2(N+1)}\right)-\omega\left(-\frac{5 \pi}{2(N+1)}\right) \\
\xi_{3}^{-1} & =\omega\left(\frac{7 \pi}{2(N+1)}\right)-\omega\left(\frac{\pi}{2(N+1)}\right)+\omega\left(-\frac{\pi}{2(N+1)}\right)-\omega\left(-\frac{7 \pi}{2(N+1)}\right) \\
& \vdots \\
\xi_{n}^{-1} & =\omega\left(\frac{(2 n+1) \pi}{2(N+1)}\right)-\omega\left(\frac{\pi}{2(N+1)}\right)+\omega\left(-\frac{\pi}{2(N+1)}\right)-\omega\left(-\frac{(2 n+1) \pi}{2(N+1)}\right)
\end{aligned}
$$

where $\omega(x)$ is given by

$$
\omega(x)=\frac{1}{2} \ln [1+\sin (4 u) \sin x]
$$

The sum in Eq. (15) can be transformed as

$$
\sum_{k=1}^{\frac{N}{2}} \omega\left(\frac{k-1 / 2}{N+1} \pi\right)=-\frac{1}{2} \omega\left(\frac{\pi}{2}\right)+\frac{1}{2} \sum_{k=0}^{N} \omega\left(\frac{k+1 / 2}{N+1} \pi\right),
$$

and can be handled by using the Euler-Maclaurin summation formula [13]. Suppose that $F(x)$ together with its derivatives is continuous within the interval $(a, b)$. Then the general Euler-Maclaurin summation formula states

$$
\sum_{n=0}^{N-1} F(a+n h+\alpha h)=\frac{1}{h} \int_{a}^{b} F(\tau) \mathrm{d} \tau+\sum_{k=1}^{\infty} \frac{h^{k-1}}{k !} \mathrm{B}_{k}(\alpha)\left(F^{(k-1)}(b)-F^{(k-1)}(a)\right)
$$

where $0 \leq \alpha \leq 1, h=(b-a) / N$ and $\mathrm{B}_{k}(\alpha)$ are so-called Bernoulli polynomials defined in terms of the Bernoulli numbers $B_{p}$ by

$$
\mathrm{B}_{k}(\alpha)=\sum_{p=0}^{k} \mathrm{~B}_{p} \frac{k !}{(k-p) ! p !} \alpha^{k-p}
$$

Indeed, $B_{n}(0)=B_{n}$. Bernoulli polynomials satisfy the identity:

$$
B_{n}(1 / 2)=\left(2^{1-n}-1\right) B_{n}
$$


Using the Euler-Maclaurin summation formula given by Eq. (22) with $a=0, b=\pi, \alpha=1 / 2$ and $F(x)=\omega(x)$ the asymptotic expansion of the critical free energy $f_{N}$ can be written in the following form

$$
\begin{aligned}
N\left(f_{N}-f_{\infty}\right) & =f_{\text {surf }}-\sum_{p=1}^{\infty} \frac{\lambda_{2 p-1}}{(2 p-1) !}\left(\frac{B_{2 p}(1 / 2)}{2 p}+2^{-2 p+2}\right)\left(\frac{\pi}{N+1}\right)^{2 p-1}, \\
& =f_{\text {surf }}-\frac{23 \pi \sin 4 u}{48(N+1)}-\frac{247\left(\sin ^{3} 4 u-\frac{1}{2} \sin 4 u\right)}{5760}\left(\frac{\pi}{N+1}\right)^{3}+\ldots,
\end{aligned}
$$

where

$$
\begin{aligned}
f_{\infty} & =\frac{1}{\pi} \int_{0}^{\pi} \omega(x) d x \\
f_{\text {surf }} & =-\frac{1}{4} \ln (1+\sin 4 u)+\frac{1}{\pi} \int_{0}^{\pi} \omega(x) d x
\end{aligned}
$$

and $\lambda_{k}$ is the coefficients in the Taylor expansion of the $\omega(x)$ :

$$
\omega(x)=\sum_{p=0}^{\infty} \frac{\lambda_{p}}{p !} x^{p},
$$

with $\lambda_{0}=0, \lambda_{1}=\frac{1}{2} \sin 4 u, \lambda_{2}=-\frac{1}{2} \sin 4 u^{2}, \lambda_{3}=\sin ^{3} 4 u-\frac{1}{2} \sin 4 u, \ldots$

Using the Taylor expansion of the $\omega(x)$ the asymptotic expansion of the critical inverse correlation lengths $\xi_{r}^{-1}$ for $r=1,2,3, \ldots, n$ can be written in the following form

$$
\begin{aligned}
\xi_{1}^{-1} & =\sum_{p=1}^{\infty} \frac{2\left(3^{2 p-1}-1\right) \lambda_{2 p-1}}{2^{2 p-1}(2 p-1) !}\left(\frac{\pi}{N+1}\right)^{2 p-1}, \\
& =\frac{\pi \sin 4 u}{N+1}+\frac{13\left(\sin ^{3} 4 u-\frac{1}{2} \sin 4 u\right)}{12}\left(\frac{\pi}{N+1}\right)^{3}+\ldots, \\
\xi_{2}^{-1} & =\sum_{p=1}^{\infty} \frac{2\left(5^{2 p-1}-1\right) \lambda_{2 p-1}}{2^{2 p-1}(2 p-1) !}\left(\frac{\pi}{N+1}\right)^{2 p-1}, \\
& =\frac{2 \pi \sin 4 u}{N+1}+\frac{31\left(\sin ^{3} 4 u-\frac{1}{2} \sin 4 u\right)}{6}\left(\frac{\pi}{N+1}\right)^{3}+\ldots, \\
\xi_{3}^{-1} & =\sum_{p=1}^{\infty} \frac{2\left(7^{2 p-1}-1\right) \lambda_{2 p-1}}{2^{2 p-1}(2 p-1) !}\left(\frac{\pi}{N+1}\right)^{2 p-1}, \\
& =\frac{3 \pi \sin 4 u}{N+1}+\frac{57\left(\sin ^{3} 4 u-\frac{1}{2} \sin 4 u\right)}{4}\left(\frac{\pi}{N+1}\right)^{3}+\ldots \\
& \vdots \\
\xi_{n}^{-1} & =\sum_{p=1}^{\infty} \frac{2\left[(2 n+1)^{2 p-1}-1\right] \lambda_{2 p-1}\left(\frac{\pi}{N+1}\right)^{2 p-1},}{2^{2 p-1}(2 p-1) !} \\
& =\frac{n \pi \sin 4 u}{N+1}+\frac{\left[(2 n+1)^{3}-1\right]\left(\sin ^{3} 4 u-\frac{1}{2} \sin 4 u\right)}{24}\left(\frac{\pi}{N+1}\right)^{3}+\ldots,
\end{aligned}
$$


Eqs. (25) and (29) - (32) imply that the ratios of amplitudes of the $(N+1)^{-(2 p-1)}$ correction

terms in the free energy and the inverse correlation lengths expansions, i.e., $b_{p}^{(n)} / a_{p}$ should not depend on the spectral parameter $u$ and given by

$$
\frac{b_{p}^{(n)}}{a_{p}}=-\frac{(2 n+1)^{2 p-1}-1}{\frac{2^{2 p-2} B_{2 p}(1 / 2)}{2 p}+1}
$$

For $p=1$ we have

$$
\frac{b_{1}^{(n)}}{a_{1}}=-\frac{48}{23} n
$$

and for $p=2$ we have

$$
\frac{b_{2}^{(n)}}{a_{2}}=-\frac{240}{247}\left[(2 n+1)^{3}-1\right]=-\frac{480}{247} n\left(4 n^{2}+6 n+3\right) .
$$

\section{PERTURBATED CONFORMAL FIELD THEORY}

The finite-size corrections to Eqs. (22) and (31) can be calculated by the means of a perturbated conformal field theory [14, 15]. In general, any lattice Hamiltonian will contain correction terms to the critical Hamiltonian $H_{c}$

$$
H=H_{c}+\sum_{p} g_{p} \int_{-N / 2}^{N / 2} \phi_{p}(v) d v
$$

where $g_{p}$ is a non-universal constant and $\phi_{p}(v)$ is a perturbative conformal field. Below we will consider the case with only one perturbative conformal field, say $\phi_{l}(v)$. Then the eigenvalues of $H$ are

$$
E_{n}=E_{n, c}+g_{l} \int_{-N / 2}^{N / 2}<n\left|\phi_{l}(v)\right| n>d v+\ldots
$$

where $E_{n, c}$ are the critical eigenvalues of $H$. The matrix element $\left\langle n\left|\phi_{l}(v)\right| n>\right.$ can be computed in terms of the universal structure constants $\left(C_{n l n}\right)$ of the operator product expansion [14]: $\left\langle n\left|\phi_{l}(v)\right| n\right\rangle=(2 \pi / N)^{x_{l}} C_{n l n}$, where $x_{l}$ is the scaling dimension of the conformal field $\phi_{l}(v)$. The energy gaps $\left(E_{n}-E_{0}\right)$ and the ground-state energy $\left(E_{0}\right)$ can be written as

$$
\begin{aligned}
E_{n}-E_{0} & =\frac{2 \pi}{N} x_{n}+2 \pi g_{l}\left(C_{n l n}-C_{0 l 0}\right)\left(\frac{2 \pi}{N}\right)^{x_{l}-1}+\ldots \\
E_{0} & =E_{0, c}+2 \pi g_{l} C_{0 l 0}\left(\frac{2 \pi}{N}\right)^{x_{l}-1}+\ldots
\end{aligned}
$$


Note, that the ground state energy $E_{0}$ and the energy gaps $\left(E_{n}-E_{0}\right)$ of a quantum spin chain are, respectively, the quantum analogies of the free energy $f_{N}$ and inverse correlation lengths $\xi_{n}^{-1}$ for the Ising model; that is,

$$
N f_{N} \Leftrightarrow-E_{0}, \quad \text { and } \quad \xi_{n}^{-1} \Leftrightarrow E_{n}-E_{0} .
$$

For the 2D Ising model, one finds [16] that the leading finite-size corrections $\left(1 / N^{3}\right)$ can be described by the Hamiltonian given by Eq. (36) with a single perturbative conformal field $\phi_{l}(v)=L_{-2}^{2}(v)$ with scaling dimension $x_{l}=4$.

In order to obtain the corrections we need the matrix elements $\left\langle n\left|L_{-2}^{2}(v)\right| n\right\rangle$, which have already been computed by Reinicke [17].

$$
<\Delta+r\left|L_{-2}^{2}\right| \Delta+r>=\left(\frac{2 \pi}{N}\right)^{4}\left[\frac{49}{11520}+(\Delta+r)\left(\Delta-\frac{5}{24}+\frac{r(2 \Delta+r)(5 \Delta+1)}{(\Delta+1)(2 \Delta+1)}\right)(41)\right.
$$

The universal structure constants $C_{2 l 2}, C_{1 l 1}$ and $C_{0 l 0}$ can be obtained from the matrix element $<n\left|L_{-2}^{2}(v)\right| n>=(2 \pi / N)^{x_{l}} C_{n l n}$, where $x_{l}=4$ is the scaling dimension of the conformal field $L_{-2}^{2}(v)$.

At the critical point the spectra of the Hamiltonian with fixed $(+-)$ boundary conditions can be understood in terms of irreducible representations $\Delta$ of a single Virasoro algebra with values of $\Delta$ is $\frac{1}{2}$.

For fixed (+-) boundary conditions the ground state $\mid 0>$ and the excited states $\mid r>$ $(r=1,2, \ldots, n)$ are given by

$$
|r>=| \Delta=\frac{1}{2}, r=k>, \quad \text { for } \quad k=1,2, \ldots, n
$$

After reaching this point, one can easily compute the universal structure constants $C_{r l r}$ $(r=0,1,2, \ldots, n)$ for fixed $(+-)$ boundary conditions. The values of $C_{r l r}$ can be obtained from Eqs. (41) and (42) and given by:

$$
\begin{aligned}
& C_{r l r}=\frac{1729}{11520}+\frac{7 r\left(4 r^{2}+6 r+3\right)}{24} \\
& C_{0 l 0}=1729 / 11520, \quad C_{1 l 1}=45409 / 11520, \quad C_{2 l 2}=210049 / 11520, \ldots
\end{aligned}
$$

Equations (38) and (39) implies that the ratios of first-order corrections amplitudes for $E_{n}-E_{0}\left(\xi_{n}^{-1}\right)$ and $-E_{0}\left(f_{N}\right)$ is universal and equal to $\left(C_{0 l 0}-C_{n l n}\right) / C_{0 l 0}$, which is consistent with Eq. (35)

$$
r_{n}(2)=\frac{C_{0 l 0}-C_{n l n}}{C_{0 l 0}}=-\frac{480}{247} n\left(4 n^{2}+6 n+3\right)
$$




\section{SUMMARY}

In this paper we present exact calculations for all coefficients in the asymptotic expansions given by Eqs. (66) and (7) for an infinite number of specific levels $E_{n}(n=0,1,2, \ldots)$, for

which we find that the ratios $b_{p}^{(n)} / a_{p}$ are indeed universal and doesn't depend on the spectral parameter $u$. We find that such universal behavior are correctly reproduced by the conformal perturbative approach.

\section{ACKNOWLEDGEMENTS}

The work of NSI is supported in part by National Center for Theoretical Sciences: Physics Division, National Taiwan University, Taipei, Taiwan and by Institute of Physics, Academia Sinica, Taipei, Taiwan.

[*] Electronic address: izmail@yerphi.am; izmailan@phys.sinica.edu.tw

[2] A. Aharony and G. Ahlers, Phys. Rev. Lett. 44, 782 (1980); M.-C. Chang and A. Houghton, ibid. 44, 785 (1980); A. Aharony and M.E. Fisher, ibid. 45, 679 (1980).

[3] N. S. Izmailian and C.-K. Hu, Phys. Rev. Lett. 86, 5160 (2001).

[4] N. S. Izmailian and C.-K. Hu, Nucl. Phys. B 808, 613 (2009).

[5] N. S. Izmailian and Y.-N. Yeh, Nucl. Phys. B 814, 573 (2009).

[6] N. S. Izmailian, Nucl. Phys. B 839, 446 (2010).

[7] D. L. O'Brien, P. A. Pearce, and S. Ole Warnaar, Physica A 228, 63 (1996).

[8] V. Privman and M. Fisher, Phys. Rev. B 30, 322 (1984).

[9] H.W.J. Blöte, J.L. Cardy, M.P. Nightingale, Phys. Rev. Lett. 56 (1986) 742.

[10] I. Affleck, Phys. Rev. Lett. 56 (1986) 746.

[11] J. Cardy, Nucl. Phys. B 275 (1986) 200.

[12] H. J. Brascamp and H. Kunz, J. Math. Phys. 15, 66 (1974).

[13] G.H. Hardy, Divergent Series, (Clarendon Press, Oxford, 1949).

[14] J. Cardy, Nucl. Phys. B 270 (1986) 186.

[15] A. B. Zamolodchikov, Sov. J. Nucl. Phys. 46 (1987) 1090. 
[16] For more details see M. Henkel, Conformal invariance and critical phenomena, (Springer Verlag, Heidelberg, 1999), Chap. 13.

[17] P. Reinicke, J. Phys. A: Math. Gen. 20 (1987) 5325. 